\title{
Essais
}

Revue interdisciplinaire d'Humanités

Hors-série 7 | 2022

Récits d'outre-thèse

\section{Le chemin de la « selva oscura » : raisonner ou se faire une raison?}

The path of the "selva oscura": Reason or self-reasoning?

\author{
Patrícia Souza Silva
}

\section{(2) OpenEdition}

Journals

Édition électronique

URL : https://journals.openedition.org/essais/10803

DOI : 10.4000/essais. 10803

ISSN : 2276-0970

Éditeur

École doctorale Montaigne Humanités

Édition imprimée

Date de publication : 15 avril 2022

ISBN : 979-10-970024-00-0

ISSN : $2417-4211$

Référence électronique

Patrícia Souza Silva, « Le chemin de la « selva oscura » : raisonner ou se faire une raison ? », Essais [En ligne], Hors-série 7 | 2022, mis en ligne le 01 février 2022, consulté le 18 janvier 2023. URL : http:// journals.openedition.org/essais/10803 ; DOI : https://doi.org/10.4000/essais.10803

Ce document a été généré automatiquement le 18 janvier 2023.

Tous droits réservés 


\section{Le chemin de la « selva oscura »: raisonner ou se faire une raison?}

The path of the "selva oscura": Reason or self-reasoning?

Patrícia Souza Silva

\section{NOTE DE L'AUTEUR}

Note sur le titre : on reconnaît ici le célèbre fragment de la Divine Comédie de Dante Alighieri, « selva oscura » en italien ou « forêt sombre » en français, où le poète entame la descente en Enfer (Inferno), en quête du « droit chemin ». Nous y reviendrons.

1 Si vous découvrez ce texte, vous êtes peut-être mort. Mais le doute existe. Si vous vous croyiez vivant, l'escalade d'une montagne vous attend. Par contre, si vous vous pressentiez en Inferno ${ }^{1}$, entamez la descente de l'enfer dantesque. Perdus, on ne sait plus si on remonte ou si on descend. Il n'y a pas de lumières, la noirceur est profonde...

2 Y aurait-il une vie après la thèse ? Notamment après une thèse ès Lettres et Sciences Humaines? Perpétuelle question. À l'arrivée au sommet, on est blessé, les chaussures détruites, les vêtements déchirés.

3 Les compliments semblent être une illusion, on est dubitatif sur ce qui est réel. La réalité ne ressemble qu'à des images défilant devant nos yeux.

4 Le cerveau ne fonctionne plus. Les yeux sont fatigués. La tête baissée n'arrive plus à se relever.

5 Reflet d'un geste devenu habituel : regarder loin, dedans, autour - regarder dans le vide. Nous qui avons gravi cette montagne, entamons la descente à quatre pattes, roulant, sur nos fesses, on n'a plus de genoux. Le crâne pèse tellement lourd, comment le porter? J'ai l'impression d'être plus aride que cette terre montagneuse, toutefois je ne suis que poussière stellaire. 
6 Je sais ce qui m'attend en bas. Il y a la vie, la ville. Les habitants ont leurs occupations, le monde est en mouvement, les enfants vont à l'école, les voitures sont véloces, les pas pressés.

7 Notre arrivée est féroce. On croise des regards familiers : «et alors, tu as réussi ?", «oui », «Félicitations!». J'ai un demi-sourire. Je n'arrive plus à parler, ces vieilles connaissances $^{2}$ me croient encore, car ils me connaissent. En dernier ressort, pour continuer mon chemin, je baisse la tête, la couvre d'une cape, rase les murs, en essayant de rentrer hâtivement chez moi, de fermer tous les volets, d'éteindre toutes les lumières, de glisser sous une couverture et de disparaître.

C'est fait. Je tremble encore.

9 La nuit est longue, je me sens amorphe. Et pourtant...il me faudrait continuer de labourer. Je sais, ce n'est pas le moment de faire une pause.

Il retentit encore des interrogations adorables, entendues au sommet même: «est-ce que ça te permettra de vivre? ». Le regard qu'on nous lance est satanique. Et pourtant, on croyait que c'était des moutons. Seraient-ils des loups? On n'a pas les idées claires. C'est le début ou la fin? Comme si je ne m'étais pas posé toutes ces questions-là auparavant. Je touche le fond. Il me faudrait peut-être un peu d'enivrement, j'ouvre le spiritueux :

Il faut être toujours ivre. Tout est là : c'est l'unique question. Pour ne pas sentir l'horrible fardeau du Temps qui brise vos épaules et vous penche vers la terre, il faut vous enivrer sans trêve.

Mais de quoi ? De vin, de poésie ou de vertu, à votre guise. Mais enivrez-vous.

Et si quelquefois, sur les marches d'un palais, sur l'herbe verte d'un fossé, dans la solitude morne de votre chambre, vous vous réveillez, l'ivresse déjà diminuée ou disparue, demandez au vent, à la vague, à l'étoile, à l'oiseau, à l'horloge, à tout ce qui fuit, à tout ce qui gémit, à tout ce qui roule, à tout ce qui chante, à tout ce qui parle, demandez quelle heure il est ; et le vent, la vague, l'étoile, l'oiseau, l'horloge, vous répondront: «Il est l'heure de s'enivrer! Pour n'être pas les esclaves martyrisés du Temps, enivrez-vous; enivrez-vous sans cesse! De vin, de poésie ou de vertu, à votre guise. $»^{3}$

11 Le poète maudit connaissait la formule pour affronter ou encore respirer la réalité. Tout ce temps, ce longtemps, à quoi servait-il vraiment ? Est-ce qu'il fallait vraiment gravir cette montagne? Je suis montée, je suis descendue, j'ai un document qui le prouve, nonobstant j'ai les mains nues, vides. Il faut se revivifier, rentrer dans les engrenages du monde. Je dois bien servir à quelque chose. Il y a des cases à remplir. "Quelle est votre activité professionnelle»? Il n'y a pas la case : « je suis docteur, mais je n'ai pas d'emploi, pas de tune, pas beaucoup d'expérience non plus ». Dans mon cas : « euh, je suis mère au foyer ». Je n'ose pas avouer que j'ai un doctorat. Un jour, dans une discussion amicale, j'ose exposer mon point de vue. C'était à Londres. Je précise que c'était à Londres, car dans le temps passé là-bas, une année, je n'ai réussi à avoir aucun emploi, même pas assistante d'école maternelle. Là-bas, il paraît que c'est l'expérience qui compte le plus. Il faut bien qu'il y ait un début, bref. Pour revenir à la discussion avec ces amis-là, après m'avoir donné plusieurs arguments basés sur l'expérience d'un tel, je dis, «attends, mais j'ai quand même un doctorat dans le domaine ». Et là...tous se taisent, devant moi, me haïssent...Je n'ai pas le droit de dire que j'ai un doctorat. Je suis en train de me vanter, pire, je suis en train d'humilier les autres. Ah oui, «tu as beaucoup changé ».

Et comment ! Si vous saviez... 
13 J'ai passé beaucoup de temps avec moi-même. J'ai sorti mon cerveau, l'ai mis sur la table et lui ai donné des coups de marteau pour voir s'il fonctionnait. Je l'ai tripoté sur les côtés, devant, derrière, je l'ai électrocuté, tapé au fer chaud...Oui, j'ai changé. La compagnie seule de la raison, de l'autonomie, de l'analyse, m'a poussée je ne sais où, oui. Et tout cela pourquoi ? À quoi ça me sert ? Pour être en compagnie de la raison, on doit être seul...Une solitude pour écrire. J'aimerais être le génie romantique possédé d'une intelligence déchaînée d'écriture, d'où des idées jailliraient continuellement... et voilà, la thèse est prête, tam dam, miracle! On arrête la rêverie, des lectures et des lectures perdues, des recherches fourvoyées, huit jours pour écrire un paragraphe, et pourquoi tout cela? Pour ne pas avoir d'expérience professionnelle?

L'autre jour, j'ai vu une annonce pour stagiaire en traduction de français-portugais. C'est exceptionnel, une telle offre! Bien, je pourrais avoir une expérience rémunérée, va savoir combien, mais rémunérée. Je n'ai pas besoin de dire le dénouement. C'était non. Et pourtant, je fais de la traduction littéraire et poétique (on oublie le financier et songe à l'honneur !). Si, j'ai fait une thèse en traduction poétique. L'envie d'un rire hystérique...

16 On ne sait plus si c'est l'humour ou la folie qui s'empare de moi. Est-ce que mon cerveau fonctionne vraiment? Combien de temps faut-il pour comprendre un poème? Et pour le décortiquer? Une thèse en traduction de poésie. Point. Points de suspension... Silence.

17 Je suis docteure en Littérature Générale et Comparée. Dans ma thèse, d'une part j'ai présenté un auteur brésilien encore inconnu du public francophone, établi la critique et l'analyse d'une œuvre qui n'avait pas encore été vraiment examinée. De ce fait, un travail de longue haleine, suivant une démarche comparatiste avec d'autres auteurs français et brésiliens. Et d'autre part, j'ai fait des recherches en traductologie pour soutenir la traduction intégrale de ce recueil de poèmes que j'ai faite (et que je compte bien sûr publier).

18 Dans l'atelier d'écriture de ma thèse, comme pour presque tous en LSHS, vivre était chronophage, livrophage, psychophage, pourquoi pas autophage. On est habitué aux thèses très volumineuses dans ce domaine, quoi qu'en traduction poétique, les caractères s'ajoutent au compte-gouttes, lentement, car avant d'être traducteur, il faut être lecteur. Solitaire, en s'appuyant sur les innombrables travaux qui reflètent la critique de ceux qui exercent ou qui étudient cet acte recréateur. Dans cette pratique, on se ressource de la traductologie, discipline énigmatique, possédant le don, parfois, d'accentuer encore plus les problèmes, soit pour y répondre soit pour se questionner sur elle-même. Par conséquent, le développement d'une traduction est accompagné d'une action et d'une réflexion fortement indissociables.

19 La traduction doit donner des couleurs, faire revivre le texte dans une nouvelle langue, et pas seulement reproduire les formes figées d'une écriture. Le texte reprend vie lors de la lecture. Dans A Casa ${ }^{4}$, il existe un conflit entre la présence des objets et leur sens, signifiant et signifié. La tâche de traduction doit aller au-delà des effets stylistiques du texte, des rimes et des sonorités, car il y a aussi une présence à reproduire. Comment créer un effet de présence d'un objet dans une autre langue que celle du poème d'origine? Il se peut que plus tard, les obstacles ressemblent à des évidences. Et peutêtre devrons-nous, une fois de plus, affronter des réinterprétations, des sonorités ignorées, une autre mise en forme plus adaptée ? Sûrement. Ma thèse est donc arrivée à 
son terme, mais sans réponse définitive, si ce n'est plus d'interrogations. L'impression est que la traduction, surtout poétique, est un acte extrêmement fragile et discutable ; tout peut basculer par la remise en question d'un seul mot. En lisant d'autres traductions, justement pour réfléchir sur cet acte, je me suis également surprise en train d'interroger le travail des autres traducteurs, hélas! Peut-être faudrait-il simplement accepter la traduction comme une matière vivante, modulable, en constante transformation?

Enfin, en souhaitant donner au lecteur francophone la possibilité de lire un texte proche de celui d'origine, j'ai dédié beaucoup de temps à des pages qui ne se multipliaient pas. Que de révisions de ce recueil de poèmes : j'en ai fait au minimum une dizaine. Et si je regarde encore, je suis sûre de toujours trouver de quoi changer encore. Pour cette raison, je me suis forcée à mettre un point final. Je sais que je n'ai pas excédé la durée moyenne de cinq années pour une thèse en LSHS. Malgré les félicitations, on se sent indigne, pire, un usurpateur, imposteur.

Maintenant, la soutenance est passée. Deux années se sont écoulées à une vitesse éclair. On est pris dans les engrenages de la machine du travail. Avant, lorsqu'on était encore doctorant, c'était bien intéressant de cocher la case étudiante et d'avoir une carte étudiante. «Ah, bon? Vous êtes étudiante »? On nous demande en fixant notre visage plus si jeune, dans mon cas, en me regardant avec une poussette. À quoi on répondait superbement: «je fais un doctorat en Littérature », «Bravo!». Désormais, lorsqu'il faut remplir des formulaires, pour quoi que ce soit, même pour postuler à la qualification, la seule option possible à cocher est : « sans activité ». Parfois, je réponds à des sondages, et là, soit «mère au foyer " soit "sans emploi, ne cherche pas de travail », car je ne suis pas inscrite à Pôle emploi. «I'm Nobody! Who are you ? ${ }^{5}$ " Que pourrais-je répondre d'autre? Écrivaine? Même si je tenais compte de mes publications sporadiques, je n'oserais pas les présenter comme une activité professionnelle. Et pourtant, j'y passe du temps, c'est une vraie occupation. On me demande, lorsque je publie, si j'ai été payée. Comment leur expliquer que toutes ces publications sont justement faites dans le but d'être payée un jour! Hélas, je ne publie pas beaucoup. Il faut reconnaître que ce n'est pas évident de concilier une vie familiale et professionnelle, surtout pour les femmes.

La doxa est persuadée que c'est tellement facile d'écrire, d'acquérir des savoirs. On nous dit que le cerveau humain est une puissante machine. J'aimerais en faire la preuve, accéder à toutes ces connaissances cumulées, débattre avec des arguments fondés sur tout ce que j'ai déjà lu depuis cinq années de double licence (portugais, anglais), deux de master et cinq de doctorat. Cependant la mémoire est faillible, la vie est-elle une constante révision? Il est vrai que lorsqu'on enseigne, on arrive à mieux se souvenir, car enseigner est aussi apprendre. Dans mon cas, chaque écrit est une constante recherche, révision de savoirs, confrontation d'idées, démarches comparatistes etc. J'avoue que je bouillonne d'idées d'articles, j'arrive à établir des liaisons entre sujets et à en déduire une ébauche théorique. Incessamment, je m'empresse de noter toutes les idées dans un cahier dédié, mais le temps est furtif. ô temps, attarde-toi sur moi, pour que je puisse écrire dans les règles de l'art. Laisse-moi devenir ton maître, car ta maîtresse, je le suis déjà !

23 Je prends beaucoup de plaisir à écrire. L'objectif professionnel serait de vivre d'une passion, c'est-à-dire transmettre et avancer des savoirs, par l'enseignement et par la recherche. Comment fait-on pour atteindre ce Graal ? Faut-il sacrifier une vie sociale et 
familiale? S'enfermer dans un laboratoire? Comme nous disait souvent une enseignante, dans le temps, le doctorat était le couronnement de toute une carrière, aujourd'hui c'est le minimum pour débuter. En avant! Je me lance. En France, un doctorat ne suffit pas pour avoir un poste, il faut la qualification. Celle-ci n'est pas la garantie d'un emploi, mais une sorte d'autorisation pour postuler. Donc, si vous voyez une offre de poste, vous ne pouvez postuler que si vous avez la qualification. Je n'approfondirai pas ce sujet, surtout qu'en ce moment j'essaie de me qualifier.

On entend sur le profil de certains candidats que ce sont des machines, que leurs curriculum vitae sont constitués de je ne sais combien de pages, d'une longue liste de publications, d'expériences etc. Motivation, où es-tu? Il semblerait qu'au tamis de la qualification, la moitié soit recalée. On s'accroche pour entamer une autre montagne et à l'arrivée du pic, il faut continuer car ce n'était qu'un passage obligatoire pour la deuxième montagne, celle aux sommets cachés dans les nuages. J'espère que dans votre domaine il existe beaucoup de postes, car en Littérature Comparée, les places sont rares. Désolée pour l'euphémisme, c'est désespérant. Et encore, si j'ai une place, où serai-je? À l'autre bout de la France? Le changement d'école pour les enfants, c'est encore acceptable, mais pour les conjoints, qui nous ont soutenus jusqu'à présent, devraient-ils quitter leur boulot? Pendant qu'ils cherchent un travail, allons-nous vivre avec le salaire initial de maître de conférences? On ne fait que jurer. On est maudit. Combien de fois va-t-on entendre encore qu'il faut assumer ses choix ? Où est-ce qu'on avait la tête?

Lorsque j'ai rencontré ma directrice de thèse, elle m'a parlé, un peu, de la rareté des postes dans l'enseignement supérieur (toujours dans notre domaine de Littérature Comparée). Mais sa bienveillance, son tact, l'envie de ne pas détruire mes rêves, font qu'elle n'a pas insisté. Vous le savez, commencer un doctorat n'est pas facile non plus. Toutefois, on se croirait dans un jeu vidéo, la seule certitude est que le prochain niveau sera plus dur. On dit que c'est la vie. D'accord, sauf que pour nous, docteurs, le chemin est plus étroit et long.

\begin{tabular}{|c|c|}
\hline $\begin{array}{l}\text { Nel mezzo del cammin di nostra vita } \\
\text { mi ritrovai per una selva oscura, } \\
\text { che la diritta via era smarrita. } \\
\text { Ahi quanto a dir qual era è cosa dura } \\
\text { esta selva selvaggia e aspra e forte } \\
\text { che nel pensier rinnova la paura! } \\
\text { tant'è amara che poco più è morte; }\end{array}$ & $\begin{array}{l}\text { Sur le milieu du chemin de la vie, } \\
\text { je me trouvai dans une forêt sombre, } \\
\text { le droit chemin se perdait, égaré. } \\
\text { Ah ! qu'il est dur de dire quelle était, } \\
\text { cette forêt sauvage, âpre et infranchissable, } \\
\text { dont le seul souvenir réveille la terreur! } \\
\text { Si amère, la mort l'est à peine un peu plus !6 }\end{array}$ \\
\hline
\end{tabular}

Le bonheur de commencer en doctorat LSHS devrait être accompagné d'un avertissement sur une grande porte: "laissez toute espérance, vous qui entrez ${ }^{7}$ ». À notre âge, toutes nos connaissances ont déjà une profession dans leur domaine. Quant à nous, c'est l'incertitude. "Pourquoi as-tu fait un doctorat?» On ne va pas recommencer! Faisons l'autruche pour continuer de rêver.

Revenons à la qualification. SI je l'ai. Je dis bien « SI » je l'ai, je disposerai de quatre ans pour conquérir un poste de Maître de Conférences. Soyons optimistes, et supposons qu'il y a cinq postes par an, donc vingt au bout de quatre années. Trop de maths, 
j'arrête le calcul. Ce serait trop beau! D'accord, continuons le raisonnement, combien de personnes ont été qualifiées cette année ? Basé sur la liste de qualifiés 2020, soixante-douze candidats ont obtenu la qualification de la CNU 10, Littérature Comparée, cette année-là. Multiplié pour les quatre années à venir $72 \times 4=288$, le nombre de qualifiés sera donc de 288 pour 20 postes, selon la logique d'une littéraire, j'espère m'être trompée. C'est encourageant ! Y a-t-il vraiment une vie après la thèse ? J'arrête d'écrire cet essai.

Il faut juste continuer. Nous qui nous dédions à la réflexion, à comprendre le monde, devons calmer nos pensées, continuer notre marche pour atteindre le sommet. Il ne faut pas s'arrêter, pour ne pas mourir de froid. Il ne faut pas s'arrêter, au risque de refroidir le sang, de ne plus avoir de veine. Je m'en veux beaucoup de parfois m'arrêter, de ne pas publier autant que les autres. Sans compter que je me demande si je ne suis pas une usurpatrice. J'ai honte parfois de dire que j'ai un doctorat. C'était peut-être pour cela qu'auparavant les gens affichaient leur diplôme ? Pour prouver aux autres et à eux-mêmes qu'ils étaient bien docteurs ? Pour se rappeler ce qu'ils avaient accompli. Ne l'oublions pas, nous avons des capacités diverses. Je le sais. Beaucoup d'endurance. Des capacités à travailler seul, à deux, en équipe, d'être leader...Une capacité à analyser, à se ressourcer, à soutenir, à débattre. Je suis sûre qu'on pourrait s'adapter à plusieurs domaines d'excellence. Il ne faut pas croire le contraire, ni accepter d'autres jugements. On trouvera bien notre place.

\section{NOTES}

1. Dante Alighieri, op. cit.

2. Je pense ici à l'essai de Sigmund Freud, "L'inquiétante étrangeté », Essais de psychanalyse appliquée, Paris, Gallimard, coll. «Idées », 1976 (1919), p. 39 : «l'inquiétante étrangeté sera cette sorte de l'effrayant qui se rattache aux choses connues depuis longtemps, et de tout temps familières. "

3. Charles Baudelaire Petits poèmes en prose, Paris, Michel Lévy frères, La Librairie Nouvelle, 1869 [1864], p. 106.

4. L'œuvre objet d'étude de ma thèse, était le recueil de poèmes d'Heleno Godoy, A casa, Goiânia, CERNE, 1992, $140 \mathrm{p}$.

5. Emily Dickinson, Poésies complètes, traduites par Françoise Delphy, Flammarion, 1955, p. 228. Traduction française : «Je suis Personne ! Qui êtes-vous?»

6. Dante Alighieri, La Divine Comédie, Traduction d'Henri Longnon, Paris, Garnier Frères, 1960, p. 11.

7. Idem, original : « Lasciate ogne speranza, voi ch'intrate ». 


\section{AUTEUR}

PATRÍCIA SOUZA SILVA

Université de Pau et des Pays de l'Adour 